\title{
Pasivos ambientales del sistema Chingaza: un análisis desde los actores y la normatividad ${ }^{1}$
}

\section{Environmental liabilities of the Chingaza system: an analysis from the actors and the regulations}

\section{Angie Geraldine Sánchez Barbón (iD}

Universidad Jorge Tadeo Lozano, Colombia

angieg.sanchezb@utadeo.edu.co

\section{Jhoan Sebastian Barajas Tarazona (D)}

Universidad Jorge Tadeo Lozano, Colombia

jhoans.barajast@gmail.com

\begin{abstract}
Resumen
La implementación de los megaproyectos para la explotación de recursos hídricos genera impactos ambientales en las zonas donde se implementan, afectando los suelos, la flora, la fauna, la sociedad y la economía. El presente estudio busca analizar los daños ambientales producidos por el sistema Chingaza, principal fuente de abastecimiento de agua para la ciudad de Bogotá. Para ello se realizó una revisión de la literatura y se aplicaron entrevistas, con el fin de identificar los daños y determinar la existencia de pasivos ambientales. Se encontró la existencia de daños en la flora y fauna; aun así, no pueden ser asociadas de forma directa al tener un factor histórico.
\end{abstract}

Palabras clave: Impactos ambientales, pasivos ambientales, sistema Chingaza.

\begin{abstract}
The implementation of megastructures for the aim of exploitation hydric resources, impact in the environment where it is implemented, affecting soil, wildlife, flora, society, and economics. In the present investigation would find analyze the environmental impacts of the Chinganza System. Chingaza is the principal supply of water to Bogotá city. We made a systematic review of literature and interviews for the identification of impacts and environmental liabilities. It's found the existence of environmental liabilities but is difficult to relate at Chingaza system for the historical impact to the community.
\end{abstract}

Keywords: environmental impacts, environmental liabilities, Chingaza System.

Articulo: Recibido el 20 de agosto de 2020 y aprobado el 15 de febrero de 2021

\section{Cómo citar este artículo:}

Sánchez Barbón, A.G. \& Barajas Tarazona J.S. 2021). Pasivos ambientales del sistema Chingaza: un análisis desde los actores y la normatividad. Reflexión política 23(47), pp. 28-40. doi: https://doi.org/10.29375/01240781.3970

1. Expresamos nuestro agradecimiento al profesor Douglas Eduardo Molina Orjuela por su apoyo. 


\section{Introducción}

Los análisis de impacto ambiental son herramientas para cuantificar los posibles daños de las empresas al ejercer sus actividades; su origen se rastrea desde 1960 con la creación de la normatividad de Estados Unidos para regular la interacción de los individuos con el medio ambiente, seguido de esto, en 1973 Canadá genera una política sobre la valoración ambiental. A partir de ese año se da una propagación de este tipo de leyes y herramientas en diferentes países, cada uno adaptándolas de acuerdo con sus necesidades y contextos ambientales. Adicionalmente, durante el mismo periodo de tiempo las empresas comenzaron a tener un papel activo al adoptar una responsabilidad social corporativa, definida como la actuación bajo unos principios éticos que las entidades establecen, adoptándolos de una manera voluntaria propositiva, con la finalidad de generar una conciencia de cuidado hacia el ambiente de manera preventiva (Vergara Tamayo \& Ortiz Motta, 2016).

Los análisis de impacto ayudan a identificar los efectos causados y generar una asignación de posibles costos para su reparación. Se busca establecer de manera detallada todos los daños producidos, siendo una tarea que en la mayoría de los casos no se puede completar en su totalidad por la complejidad, debido a que, con frecuencia, se producen efectos dominó de larga duración. La información aportada por estos estudios debe ser utilizada para el diseño de políticas que tengan como objetivo la prevención y protección de los ecosistemas, cuando las leyes fallan en su diseño, para la implementación o vigilancia, se pueden establecer daños que perduran en el tiempo, denominados pasivos ambientales (Pérez-Rincón, 2018).

El presente documento busca estudiar la explotación de los recursos hídricos tomando el caso del sistema Chingaza, cuyo objetivo principal es el abastecimiento de agua potable para la ciudad de Bogotá Colombia, el cual se establece desde 1969 a cargo de la Empresa de Acueducto y Alcantarillado de Bogotá (EAB-ESP), con planes de generar una ampliación que se encuentra en tramites desde el año 2016. Se presenta un proceso extractivo de agua de diferentes fuentes hídricas ubicadas en Cundinamarca y Meta para el abastecimiento de la represa.

Todo proceso de explotación de un recurso natural deja unos impactos ambientales en el suelo, la flora, la fauna, etc., que deben ser resarcidos, en caso de que esto no se dé, se crea un pasivo ambiental. Para no llegar a esos puntos es importante contar con una legislación adecuada y organismos de control activo.

\section{Materiales y métodos.}

La investigación es de tipo cualitativo fenomenológica, de corte transversal, con un horizonte de estudio entre 1969 y 2020.

Pregunta de investigación. ¿Cuáles son las afectaciones ambientales producidas por la implementación, desarrollo y procesos extractivos del sistema Chingaza?

Hipótesis nula. La normatividad colombiana sobre la utilización de los recursos hídricos impide la creación de pasivos ambientales en el sistema Chingaza.

Hipótesis alterna. La falta de una normatividad más específica ha generado que en el sistema Chingaza se presenten impactos ambientales que no han sido tratados de manera adecuada por la Empresa de Acueducto y Alcantarillado de Bogotá (EAB-ESP) convirtiéndolos en pasivos ambientales.

Método. Se llevó a cabo una indagación sistemática de la literatura, se usó como motor de búsqueda Google, donde se identificaron estudios ambientales sobre el sistema Chingaza, así como proyectos establecidos por EAB-ESP para su recuperación. Finalmente, se establecieron entrevistas semiestructuradas de una duración aproximada de una hora, las cuales se llevaron a cabo de forma virtual a diferentes agentes: personas que trabajaron para EAB-ESP, beneficiarios de los proyectos y población afectada. Los datos obtenidos se procesaron con el programa Atlas.TI, citados bajo la decodificación ESC01, ESC02, ESC03 y ESC04 nombres asignados de forma aleatoria con el fin de proteger su identidad. 


\section{Legislación sistema Chingaza}

La principal normatividad sobre el tratamiento y manejo del recurso hídrico se rastrea hasta 1978 con el Decreto 1541 expedido por el presidente de la República de Colombia el 26 de julio, se reglamenta el dominio y uso de las aguas, cauces y riberas. Se exalta en particular para el parque Chingaza los artículos 56 y 58 , es uno de los mecanismos de protección el asignar como ente de control y vigilancia a la Unidad Administrativa Especial del Sistema de Parques Nacionales Naturales, quien realiza visitas oculares con intervención de personal idóneo en las disciplinas relacionadas. $\mathrm{El}$ artículo 58 indica que se deberán verificar las posibles afectaciones de las poblaciones que realicen aprovechamiento de aguas para menesteres domésticos.

Con el decreto 1753 de 1994 el Ministerio de Medio Ambiente en el artículo 38 indica que la autoridad ambiental competente podrá exigir la presentación de planes de manejo, recuperación o restauración ambiental a todos aquellos proyectos que iniciaron antes de la expedición de la ley 99 de 1993. En 1997 se dio respuesta a la ampliación del sistema Chingaza interpuesto por el acueducto de Bogotá en 1996 mediante Auto n. ${ }^{\circ} 314$ del 7 de mayo de 1997; la Subdirección de Ordenación y Evaluación Ambiental estableció la necesidad de la realización de un plan de manejo ambiental para evaluar la viabilidad de la ampliación.

El 31 de agosto del 2004 el Ministerio de Ambiente, Vivienda y Desarrollo Territorial Unidad Administrativa Especial Del Sistema De Parques Nacionales Naturales expidió la resolución n. ${ }^{\circ} 158$ en la cual se da respuesta positiva a las concesiones de aguas sobre las Quebradas El Mangón, El Mangoncito y la Chupadera, interpuesta por el acueducto de Bogotá el 5 de septiembre del 2000, con el fin de establecer los caudales ecológicos y sociales, se especificó un caudal para el río Guatiquía de $0.562 \mathrm{~m} 3 / \mathrm{s}$, río Chuza de $0.537 \mathrm{~m} 3 / \mathrm{s}$ y Guebrada Leticia de $0.030 \mathrm{~m} 3 / \mathrm{s}$, se hace la salvedad de que se debe contar con las herramientas necesarias para poder realizar un seguimiento a estos caudales. Este plan fue aprobado por las Corporaciones Autónomas Regionales (CAR) por medio del comunicado UPDIG-CJU 00006171 de noviembre 27 de 2003, el cual tendría vigilancia por parte de la Unidad Administrativa Especial del Sistema de Parques Nacionales Naturales, con el fin de tener un control e intervención en el flujo de los cauces.
En la resolución 030 de 2007 se expide el plan de manejo del Parque Nacional Natural Chingaza (PNNC), se plantea la protección del hábitat necesario para especies amenazadas, endémicas y migratorias de alta montaña, siendo la especie de mayor relevancia el oso de anteojos. Durante el plan no se especifica qué medidas le corresponderá al EAB-ESP, solo se indica que este debe acatar e implementar los proyectos que le indique el parque; al tiempo que tiene la potestad de realizar aportes de manera voluntaria siempre y cuando se aprueben previamente. El 06 de mayo de 2016, el Ministerio de Ambiente y Desarrollo Sostenible delimitó el páramo de Chingaza por medio de la resolución 0710, confirmó su existencia en 24 municipios con una extensión de 111.667 hectáreas (ha), además se decreta la prohibición de actividades de exploración y/o explotación de recursos naturales no renovables, se incluyen directrices específicas de las actividades agropecuarias, manejos y usos de estas áreas, se confirma que deberán tener un debido seguimiento y monitoreo por parte de las corporaciones autónomas.

\section{Impactos del sistema Chingaza}

Una parte significativa de las construcciones del sistema Chingaza se ubican en el PNNC cuya biogeografía se clasifica como páramo y superpáramo, caracterizado por tener en las zonas de mayor altura una vegetación predominante de gramíneas y frailejones, acompañados de formaciones arbustivas o manchas de bosque altoandino, compuesto por Encendillo, Rodaminte, Colorado, Compuestas, Ericaceae, etc., mientras en las zonas bajas se observan arbustos y pastizales azules que no son de consumo animal (Van der Hammen, Pabón, Gutiérrez, \& Alarcón, 2002).

El sistema se construye en el periodo comprendido entre 1969 y 1973, cuatro años en los que se generó la creación de la infraestructura básica para su funcionamiento (represa, tuberías, puntos de control, etc.), además del establecimiento de caminos para facilitar el acceso de materiales $\mathrm{y}$ trabajadores para su edificación $\mathrm{y}$ futuro mantenimiento. Estos procesos generan un impacto negativo en los ecosistemas al interrumpir el hábitat y modificarlo, alterando así las conductas de las especies nativas, como es el caso de los animales que se ven expuestos a una mayor interacción con los seres humanos y nuevos tipos de contaminación como la auditiva dado el uso de maquinarias (Pretelt, 2013). 
En el proceso de entrevistas la persona ESC03, al hablar sobre los métodos de mantenimiento, manifestó que:

Durante los procesos de reparación de los túneles vierten aceites dentro de estos, que al ser mezclados con el agua se vuelven tóxicos para los animales, estos consumen esta agua y mueren. También cuando descargan agua en ocasiones se crece tanto la cuenca que se lleva a los animales.

Esto indica que la fauna de la zona se ve en peligro debido a la contaminación que se genera por los procesos diarios para el funcionamiento del sistema, dado que interviene directamente en las fuentes hídricas vitales para la supervivencia de las especies de la zona. Otro ejemplo de esta situación es el proceso de descargue de aguas, el cual tiene un fuerte impacto auditivo que produce que las especies nativas tengan que emigrar.

Hasta este punto se han mencionado algunos de los efectos directos asociados al sistema; sin embargo, también existen algunos indirectos como lo manifestó el individuo ESC02: "Se ha mejorado la flora y fauna en el parque, pero no en el sistema, solo en las zonas aledañas”. Aunque la declaración puede ser un poco confusa, se indica que en el punto donde se regula el suministro de agua hacia San Rafael se presenta constantemente tráfico pesado de volquetas y de maquinaria, es usual que los conductores y los trabajadores alimenten a los animales, lo que genera adaptabilidad de estas especies a los humanos adquiriendo dietas poco saludables. Esto lleva a concluir que la entidad de Parques Nacionales Naturales de Colombia juega el papel principal respecto a la protección y recuperación de los animales, pero en las zonas que están fuera de su jurisdicción se ve poca intervención.

Uno de los mayores peligros al que se enfrenta el sistema Chingaza es la desecación y disminución del caudal, como sucede en uno de los tramos del río Guataquía que nutre el sistema, impactando en la vegetación y animales que se sustentan de esta fuente hídrica como los osos de anteojos, venados de cola y colorados, pumas, tigrillos, chivo de páramo, zorros, cóndores, águilas, gallinetas azules, entre otros; varias de ellos clasificadas en peligro de extinción (Pretelt, 2013). Esta situación se puede comprobar en los estudios realizados por parte de la CAR, donde se demuestra que el caudal de los ríos no logra mantenerse y presentan una tendencia a disminuir, en los últimos años se presenta mayor gravedad debido a factores externos como la urbanización de las zonas aledañas a la ribera, además de la mala utilización de los recursos. Dos ejemplos de esto es la ausencia de plantas de tratamientos para el vertimiento de desechos a los ríos y una sobreutilización del recurso por parte de la ganadería.

Se determina como uno de los principales factores de riesgo para el ecosistema del PNNC las poblaciones aledañas, por las prácticas económicas desempeñadas caracterizadas por tener un factor cultural o histórico.

La población aledaña al PNNC se ha caracterizado desde el siglo XIX por enfocarse en la agricultura. Entre sus principales productos se encuentran la papa, arveja, maíz y haba, además de la producción de leche debido a su actividad ganadera (Morales M., et al., 2007).

Entre 1970 y 1990 se encuentran estudios sobre el estado de los páramos de Colombia, uno de los que toma relevancia por su objeto de estudio es el realizado en Laguna Verde, Cundinamarca; el cual, cuenta con unas características y datos similares al Parque Natural Chingaza del que, por el contrario, no se disponía de mucha información para la época. Desde 1970 los páramos se encontraban en alto grado de vulnerabilidad por las prácticas agrícolas, debido a la alta productividad de la tierra se hicieron procesos de quemas de la vegetación nativa para sustituirlas principalmente por cultivos de papa y pastizales. Durante los 20 años que se realizó seguimiento se dio un decremento de la vegetación nativa del 94\%. Finalmente, se estimó que los impactos producidos serían de larga perturbación (Cárdenas, 2013; Van der Hammen et al., 2002).

Para el año 2013, el páramo Chingaza, en su totalidad de extensión, abarcaba el 23,09\% del bosque andino; mientras que la vegetación del superpáramo solo cubría un 3,14\% de su territorio. El resto de territorio mantenía la vegetación que se había introducido artificialmente por parte de los habitantes del sector para desempeñar sus actividades económicas. Es de resaltar que para ese periodo la población poco acataba la normatividad de protección del parque, por lo que podían encontrarse asentamientos para su vivienda y actividades de cultivo, especialmente para la papa y el maíz (Empresa de Acueducto Alcantarillado y Aseo de Bogotá \& Fundación para Desarrollo Sostenible Territorial, 2013). Estos datos concuerdan con los hallazgos de la CAR, donde se identifica que, previo al establecimiento del sistema Chingaza, se presentaban un fuerte daño ambiental por parte de los habitantes. Como 
consecuencia, se dificulta estimar la existencia de pasivos ambientales respecto a la flora y fauna, dados dos motivos: ausencia de datos sobre el ecosistema e impactos de larga perturbación por las prácticas de agricultura y ganadería.

A pesar de los estudios, la comunidad no está de acuerdo y sostiene que una de las principales causas de los daños ambientales se da por la irregularidad en el caudal y modificaciones en el terreno, y no por factores históricos; argumentan que la implementación de una represa genera cambios en los ecosistemas fluviales al rediseñar el sistema hídrico regional con implicaciones más allá de las locales (Pelayo \& Gasca, 2019).

Al establecer que el afluente natural se modifica, este es regulado por el sistema, el cual debe velar por el mantenimiento de unos mínimos y máximos aceptables ecológicos, pero cuando se genera un incumplimiento de estos se producen daños; debido a los factores mencionados previamente, los más fáciles de cuantificar son los sociales.

El 12 de julio de 1997 se presentó una creciente en el caudal, lo que generó el rompimiento de los diques provocando inundaciones, el principal responsable fueron las descargas producidas por la represa de Chuza de acuerdo con la comunidad. La EAB-ESP no asumió responsabilidad alguna ante lo acontecido, en su lugar, optó por excusarse diciendo que se originó por las lluvias; de esta forma, omitieron su deber como ente regulador del caudal. Según su posición, la EAB-ESP debió tener planes de contingencia para dichos hechos, los cuales por su ausencia constituyen una violación a los acuerdos pactados en la resolución 1418 de 2004, donde se indica que la empresa, al tener que realizar evacuaciones de agua no programadas, tienen la responsabilidad de avisar a diferentes organismos la situación y así tener la capacidad de determinar el impacto de la situación y plantear posibles soluciones. Sin embargo; de acuerdo con los testimonios de la comunidad, dichas evaluaciones no eran realizadas, ya que era usual presentar inundación en varios sectores y épocas del año, una de las últimas fue documentada en el 2008 en el aeropuerto de Villavicencio (Redacción EL TIEMPO, 1997; 2008).

Respecto a la ganadería, la población indica que es una actividad que ha ido decreciendo por el establecimiento del sistema. Como ejemplo señalaron la situación de la vereda El Rodeo, la cual tenía una fuerte tradición campesina; pero, debido a las limitaciones del uso del agua impuestas por Chingaza, la mayoría de los individuos tuvieron que abandonar sus actividades económicas y migrar. Los que lograron permanecer fueron aquellos con la capacidad de adquirir un punto de agua con un costo aproximado entre 15 y 20 millones de pesos y, además, cambiaron su fuente de ingresos al convertir sus hogares en hospedajes. De acuerdo con ES02, para la comunidad "se generó un desplazamiento forzado a la población de pocos recursos".

En el contexto de estos hechos, la comunidad de este lugar se percibe como víctima de desplazamiento; no solo por la creación del sistema, sino también por las prácticas por fuera de la ley que han utilizado la EAB-ESP. En diferentes zonas el terreno se volvió inestable durante el proceso de construcción por la vibración, lo que obligó a varias familias a dejar sus casas; tras varios años, algunos volvieron y encontraron sus terrenos ocupados con infraestructura del sistema Chingaza e impidiéndoles el ingreso por parte del acueducto de Bogotá, el cual no realizó una compra formal de los terrenos y tampoco dio una remuneración económica por los daños causados.

Donde se establecieron las bocatomas de los túneles, las comunidades les hacían la guerra de frente, los trataron de desplazar. Hasta lastimaron a una señora, trataron de cambiar los documentos de la extensión de la quebrada para reducir sus terrenos, pero ella guardó las escrituras en Bogotá para tener las evidencias de la extensión de sus tierras. Sobre esta problemática se realizó un documental. (ESCO2).

Los habitantes tienen la sensación de estar en un campo de batalla, en cualquier momento pueden esperar repercusiones violentas por parte del EAB-ESP; incluso anticipan la posibilidad de que alteren documentos legales para perjudicarles, tal y como lo narran en el caso anterior de lo sucedido con los terreros de la señora.

Se denota un sentimiento de inconformidad de la comunidad hacia el acueducto de Bogotá, porque se sienten maltratados y se enfrentan constantemente a injusticias, esto se observa por la falta de compensación durante 35 años y, que como indicó ESC03, "Deben pagar el impuesto predial donde pasa el tubo, pero no lo pueden usar, la gente ha demandado, algunos les ha ido bien otros mal o dejan de esa manera”. Se presenta una inconformidad sobre el impuesto del predio ya que el acueducto es el que le da utilización y mantiene vigilancia en la zona para impedir el uso de los habitantes de esas tierras, que consideran tener el derecho de dar uso a esos terrenos. 
La construcción del sistema Chingaza ha generado conflictos sociales de gravedad; un ejemplo de esto sucedió en una de las veredas cercanas, en donde la tierra tapó las vías de acceso como consecuencia de los procesos de descargue de agua, ya que con estas acciones se producen fuertes vibraciones que terminan colapsando la tierra y que caiga sobre las carreteras. "la vibración generada por las descargas produce como si fuera una detonación fuerte y desestabiliza el terreno" (ESC03). Adicionalmente, sobre los terrenos se han presentado diversos problemas como se puede observar a continuación:

"Sobre la compensación sobre los terrenos en unos casos no respondieron por el dinero de las tierras, han pasado dos generaciones que siguen luchando por los dineros" (ESC03).

Declara ESC03 que en los periodos de la construcción se llegaron a unos acuerdos por los daños que se ocasionarían sobre el valor del $20 \%$ del terreno, aproximadamente, pero estos no han sido pagados. En la vereda Buenos Aires, en la época de noviembre y diciembre, tienen desabastecimiento del agua, debido a que las pequeñas quebradas que les suministran aguas durante esos periodos son absorbidas por el sistema. "Están en el páramo y no tienen agua" (ESC03).

Desde el punto de vista de conservación y recuperación ambiental, se considera quelos diferentes asentamientos son los que producen el mayor daño en la flora y fauna, lo que ha producido fuertes choques entre EAB-ESP, el PNNC y las diferentes comunidades aledañas, siendo uno de los más notorio el apartado económico, como lo indicó ESC01, trabajador del PNNC en la línea de monitoreo e investigación de la fauna. Respecto a la situación de las familias indica que ya solo quedan dos hogares que habitan en la zona interna de la reserva, y que estas aún conservan algunas prácticas, “cazan Borugos porque no tienen más opciones”, denotando la precariedad económica de los pobladores que permanecen en la zona. Dado el mantenimiento de comportamientos que no son sustentables es importante dar incentivos económicos y otro tipo de oportunidades para poder abandonar dichas actividades de forma definitiva.

Una muestra de que este tipo de objetivos son viables es la limitación que tuvieron dos grandes causantes de los daños ambientales: la ganadería y la agricultura. Estas fueron reemplazadas en gran medida por el ecoturismo, se abrió paso al ceder algunos espacios y centralizar la comunidad bajo la figura Corpochingaza; con este nuevo proyecto diferentes personas que viven en las cercanias participan y dan una alternativa de sustento económico a las personas que allí habitan. Adicionalmente, la EAB-ESP contrata a personas del sector para que se desempeñen como guardabosques y velen por la protección del ecosistema.

El segundo individuo identificado con el código ESCO2 ha trabajado en el PNNC en la modalidad de turismo con una experiencia de diez años, además es un habitante de la zona. Manifiesta que la reserva ha mantenido, desde su punto de vista, los objetivos de preservación, conservación e investigación. Al preguntar sobre la EAB-ESP respondió:

Están acaparando los páramos, en la historia de Guasca se cuenta que se tuvieron conflictos fuertes, y pues básicamente el acueducto ha apoyado varios proyectos en la región, el apoyo ha sido mínimo, tenemos un problema con las regalías, un ejemplo es en el 2015 con lo de Bogotá humana, debería haber unas regalías a la región, bajo la modalidad de Bogotá rural, pero solo se entregó una parte del dinero, la otra no la desembolsaron. En Trinidad, sector de San Francisco, montaron un acueducto, pero no era muy bueno, y de todos modos hicieron firmar una gran cantidad de documentos a pesar de la inconformidad. Sobre el ecoturismo se recibió una ayuda para el establecimiento de una infraestructura que ellos construyeron, pero el parque no la aceptó, entonces la rechazamos, pero insisten en que se debe aceptar y ahora buscan quien lo haga posible desde la alcaldía de Guasca. (ESC02).

Los habitantes del sector tienen una sensación de desprotección al sentir que la EAB-ESP no está siendo vigilada de una manera adecuada. "La alcaldía no tiene una presencia muy fuerte, ya las demandas son por cuenta propia y con abogados privados" (ESCO2). El hecho de que las personas tengan que utilizar sus recursos económicos para poder establecer mecanismos legales genera que solo aquellos que puedan permitirse estos costos logren alzar sus voces, pero en el caso de tener pocos ingresos las opciones de las que disponen son limitadas.

Respecto a la parte ambiental, se da un sentimiento de mejora y de recuperación del ecosistema por parte de todos los entrevistados, se aclara que esto ha sido posible únicamente por la intervención del PNNC, debido a que ha impuesto una gran presión para la recuperación de la flora y la fauna, al tiempo que respeta los derechos de la población; logrando así establecer un equilibrio 
adecuado, un ejemplo de esta situación se da en los proyectos de forestación que han tenido una notable aceptación por parte de la comunidad, al brindar oportunidades en las diferentes fincas para la disminución de la actividad ganadera, y con el apoyo de campañas educativas centradas en la población infantil. Con el paso del tiempo, las personas han sido más conscientes de la riqueza que disponen y han aprendido a valorarla, logrando identificar y entender los daños ambientales que podrían acarrear un segundo proceso de ampliación del sistema Chingaza.
$\mathrm{Al}$ analizar las especies de animales que habitan en el parque, uno de los indicadores para demostrar la recuperación es un mayor número de avistamientos. Uno de los trabajadores suministró la imagen 1, indicó que en el tiempo que lleva trabajando para el PNNC ha podido observar con mayor frecuencia la fauna nativa, asocia este éxito a la creación de corredores ambientales. Sobre este aspecto es importante generar una base de datos para poder generar análisis a través del tiempo.

Imagen 1. Oso de anteojos y venado.

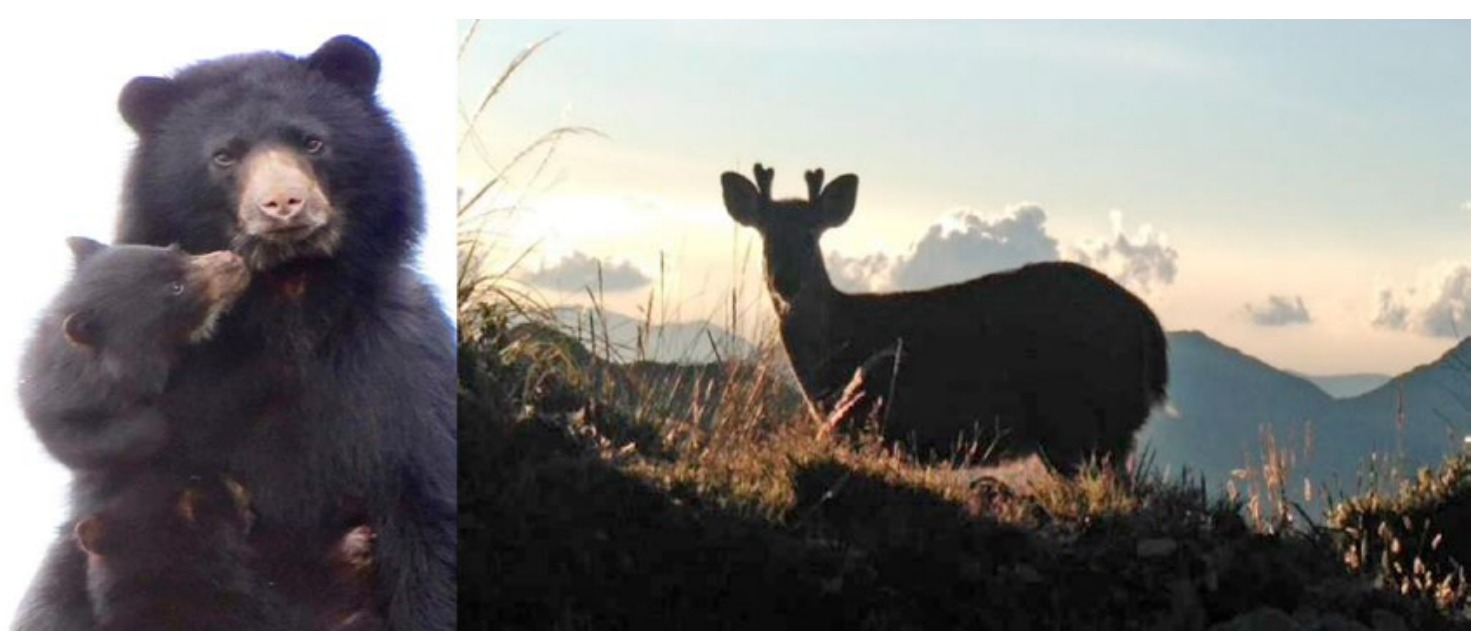

Fuente: Tomada por Adin Muñoz, 10 y 12 de octubre 2019. Lugar: Laguna Seca PNN Chingaza.

El individuo identificado con el código ESC04 es un habitante del sector de la Calera, quien ha tenido un acercamiento e interacción continua con el parque Chingaza; manifiesta que:

"las afectaciones de este lugar se están dando de dos maneras, unas asociadas con el uso y consumo del recurso hídrico y otra por las limitaciones que tienen los habitantes que tienen los predios cercanos al parque" (ESC04).

Las limitaciones que se presentan en los terrenos son producto de la clasificación catastral, al ser categorizados como zonas de amortiguación, donde solo se pueden desempeñar actividades de preservación y recuperación ambiental. Adicionalmente, indica que la problemática del parque Chingaza es que:

tiene una doble finalidad, por un lado, es un sistema abastecedor de agua y por otro lado se realiza la actividad de recreación pasiva paisajista, con presencia humana o antrópica lo cual genera una huella ecológica sobre los frailejones, sobre los ríos, sobre los líquenes, generando impactos sobre la reserva forestal. (ESCO4).

Las actividades turísticas brindan una oportunidad económica; del mismo modo, asocia unos riesgos, al presentar un impacto en el ecosistema por parte de los visitantes, además de los cambios que se producen por la adaptación de caminos e infraestructura.

Finalmente, en el año 2016 se generó un proyecto para implementar una ampliación del sistema Chingaza, buscando abastecer la creciente demanda del agua por parte de Bogotá, los conflictos entre la comunidad y la EAB-ESP se incrementaron. Uno de los principales argumentos por parte de los habitantes de la zona es su preocupación por los impactos ambientales de un nuevo proceso de construcción, conllevando a un decremento del páramo, lo que significaría ir en 
contra de las políticas de conservación.

A pesar de que la comunidad ha hecho notar su inconformidad sobre el proceso de ampliación, argumentan que no se ha dado un proceso adecuado al no poder tener una participación activa, un comportamiento similar a la primera fase del sistema Chingaza.

Ramiro Jiménez, alcalde de San Juanito, en una entrevista para el diario La Economía, manifestó que ninguna comunidad había tenido voz en los procesos para establecer el sistema Chingaza, al indicar que no se hizo un proceso de consulta pública y tampoco se especificaron los beneficios económicos de compensación (Núñez, 2016).

Aquí no tienen en cuenta los daños y como si no fuésemos parte de Colombia nos pasan por alto, no socializan nada y no presentan nada, luego aquí hay abuso y falta de sentido común porque uno no hace una casa en el lote del vecino para arrendarla, esto es arbitrario, grosero y pésimamente manejado porque todo se hace soterradamente sin decirle nada a nadie. Eso no es así, entre otras cosas porque somos dueños de la laguna de Chingaza y otros afluentes que le llevan el costoso servicio a Bogotá. (Núñez, 2016).

En una entrevista realizada por el periódico el Tiempo realizada el 12 de enero del 2019, el mandatario de Fomeque Jorge Torres: "hoy solo tenemos agua potable para el casco urbano y para 7 de las 33 veredas". Desde la percepción de la comunidad, uno de los principales culpables es el sistema de Chingaza, al generar un monopolio sobre el agua, el cual establece unos caudales que no son acordes a la demanda de los municipios, este problema se agudiza por las prácticas ganaderas y agropecuarias que dan un uso no sustentable al recurso.

Sobre la posible ampliación del sistema, las comunidades son conscientes de los posibles daños debido a todas las campañas implementadas en los últimos años para la conservación del agua y del páramo.

"El daño que hará es grande y la compensación será mínima, son pañitos de agua fría para el daño que se ocasionará y se ocasionaron" (ESCO1).

Colombia, en su Plan de Desarrollo 2018-2022, estableció como una de sus metas la equidad para el acceso al agua, pero según la población y algunos estudios, se manifiesta una inequidad inherente en el servicio, se presenta una centralización por parte de Bogotá del recurso hídrico de Cundinamarca y Meta. El acaparamiento del agua limita las actividades económicas, expone a las personas al consumo de aguas contaminadas e incentiva la migración (Herrera, 2019).

\section{Responsabilidad del Acueducto de Bogotá}

En el apartado anterior se hizo una aproximación de los daños y conflictos que se han creado en torno al sistema, en este se hace un pequeño resumen de los proyectos que ha desarrollado la EAB-ESP para reparar, prevenir y conservar.

En el año 2015 se desarrolló el proyecto "somos páramo, somos comunidad". Donde se intervino en la población que vive a los alrededores del PNNC. Se identificó como una de las principales problemáticas la escasez del agua por mala práctica en la reforestación al incorporar especies no endémicas como el eucalipto; además de esto, las fuentes hídricas a las que tiene acceso la comunidad se encuentran contaminadas como es el caso de Junín. Para solucionar esta problemática, el EAB-ESP brindó capacitaciones a los pobladores, además de la instalación de baños sanitarios y filtros en la vereda de El Valle, el cual fue calificado por ellos mismos como insuficiente si no se lograba una participación activa entre diferentes entidades (Bogotá, 2015).

Otro ejemplo se da en la vereda Guavio, que después de realizar estudios se establece como la población con el mayor impacto directo por la construcción de la represa de Chuza, su principal suministro de agua es la quebrada Montoque, la cual se ve amenazada por las malas prácticas en los cultivos, donde los residuos químicos son vertidos a esta. La intervención por parte del acueducto ha sido educativa.

Uno de los mejores ejemplos sobre las problemáticas y proyectos desarrollados se presenta en Guasca, el cual incorpora la mayoría de las problemáticas que se presentan en las zonas aledañas del parque natural. Entre 1980 y 1990, los recursos hídricos presentaron fuertes variaciones negativas en el flujo, acercándose a la escasez, generando que las personas cambien sus estilos de vida. El primero es el cambio de las formas de explotación agrícolas y ganaderas tradicionales 
que empiezan a disminuir y son reemplazadas por técnicas más modernas, o la adopción de la minería como medio de sustento. En el caso de la minería no se dio un control adecuado por parte del Estado a pesar de estar tramitando licencias ambientales, lo que conllevó a serios daños ambientales, teniendo que actuar la CAR, cerrándolas en 1991, pero el daño ya era muy grande (Cubillos, 1991).

Antes de 1970, en la región se daba por prácticas culturales una caza indiscriminada de varias especies, entre ellas el oso de anteojos. Incluso el páramo se vio fuertemente afectado por las prácticas de ganadería irresponsable, al dejar animales en libertad que destruyeron la vegetación; del mismo modo, se dieron procesos de deforestación con fines diversos (Garavito González et al., 2018).

El proyecto páramos interviene en estas poblaciones, donde su principal problemática es el agua, suministra filtros, programas de recolección de lluvias para el uso productivo en cultivos y ganadería, todo esto apoyado con una participación de la comunidad, a través de la educación se busca motivarlos a abandonar las malas prácticas (Bogotá, 2015).

Uno de los últimos proyectos implementados por la empresa del acueducto de Bogotá se centró en la biodiversidad y servicios ecosistémicos en la alta montaña (Herrera A., et al., 2015). Con la implementación de actividades educativas a diferentes grupos poblacionales pertenecientes a primaria, dando conceptos sobre la fauna y flora del lugar, además de mostrar la importancia del sistema. Durante este mismo año se realizó el contrato 00395, con el enfoque de ayudar o incentivar el incremento del turismo de la región de Suasie-Guasca. Siguiendo las directrices, el plan distrital de Bogotá 2012-2016 denominado "Bogotá Humana", la empresa EAB-ESP, con el apoyo de la secretaría distrital de planeación y la secretaría distrital ambiental, estuvieron a cargo del diseño del proyecto el cual nombraron "Conservación, restauración y uso sostenible de servicios ecosistémicos entre los páramos de Guerrero, Chingaza, Sumapaz, los Cerros Orientales y su área de influencia”, cuya ejecución se asignó a la EAB-ESP.

Tras un proceso de selección se escogió como una de las entidades beneficiarias del proyecto la Asociación Ecoturística y de Educación Ambiental
Suasie, establecida en Guasca, Cundinamarca, donde se enfocan en la protección, conservación, recuperación de recursos del medioambiente, adelantando programas de reforestación y restauración ecológica en ecosistemas afectados por desastres naturales; además el fortalecimiento, desarrollo, prevención y divulgación de actividades de ecoturismo y agroturismo, con el fin de aumentar el nivel de vida, creando en la comunidad la mentalidad y la actitud frente a los valores ecológicos.

Entre las construcciones más resaltables, se propuso la adecuación de un sendero hacia las lagunas de Siecha, el beneficio fue para la organización Suasie, la cual tendría la exclusividad para realizar los recorridos guiados. Adicional a esto, las personas a cargo del proyecto detectaron falencias de conocimiento por parte de la organización, por lo cual suministraron capacitaciones en diferentes días de noviembre y diciembre del 2015, enfocada en temas de marketing virtual, aspectos legales, contables, financieros y tributarios para ONG'S, aproximación al patrimonio cultural y plan de negocios, cada una con una duración aproximada entre dos y cuatro horas.

Finalmente, otro proyecto implementado por EAB-ESP se rastreó al año 2018 donde se realizó el proceso de contratación para realizar obras civiles para la implementación de los diseños de adecuación de tres iniciativas de turismo de naturaleza comunitaria en el marco del proyecto de conservación, restauración y usos sostenibles de servicios ecosistémicos entre los páramos de Guerrero, Chingaza, Sumapaz, los cerros orientales y su área de influencia, identificada con el código IA649-2018. Aun así, sobre este proyecto el individuo entrevistado no manifestó conformidad al indicar que no se cumplió ninguno de los objetivos que les plantearon:

Diseñar un programa de formación - capacitación para las personas vinculadas al funcionamiento de las iniciativas en marcha, diseñar bases de interpretación para los productos principales de acuerdo a la vocación de turismo de naturaleza específicamente en turismo científico- investigativo $\mathrm{y}$ ecoturismo, y turismo cultural, diseñar un manual de uso y reglamentación para los servicios y actividades del turismo de naturaleza y cultural en las veredas del municipio de Guasca, diseñar un modelo de restauración paisajística y ecosistémica 
en las áreas de los atractivos principales, diseñar dos herramientas comunicativas para la iniciativa, diseñar la infraestructura y mobiliario de instalaciones locativas blandas y bioclimáticas requeridas para la prestación de servicios en senderos y predios, diseñar la señalética de bajo impacto que guarde relación con los diseños propuestos requerida en los diferentes atractivos, diseñar los lineamientos para los planes de negocios para el proyecto ecoturístico comunitario Suasie. Aplicar la metodología para los cálculos de capacidad de carga para el sendero a las lagunas de Siecha, Parque Nacional Natural Chingaza. Realizar cuatro eventos temáticos para el desarrollo del proyecto ecoturístico comunitario Suasie, de acuerdo con las necesidades observadas durante el diagnóstico empresarial realizado. Entregar un Plan de Acción Detallado de la iniciativa. (ESC02).

Entre sus argumentos se sustenta el costo de la capacitación para cinco personas, no se recibió debido a que por persona corresponden un promedio de veintitrés millones de pesos, además se calculaba una capacitación de 1.250 horas, de las cuales en las listas de asistencia y las pruebas entregadas se alcanza tan solo un máximo de veinte horas. Además, entre las obras que entrega el acueducto, no cumplen con los requisitos del PNNC, por lo cual ha decidido no aceptarlas y actualmente se encuentran en trámite de procesos legales.

El tercer individuo ESCO3 es una persona que ha tenido la oportunidad de trabajar en los proyectos de retribución y a su vez es habitante de la zona. Manifiesta que se logra observar un descontento de la comunidad sobre EAB-ESP, dado que consideran que esta entidad solo realiza lo necesario y de una manera regular o deficiente para sus necesidades, con el único objetivo de demostrar que están cumpliendo, en este aspecto falta una mayor vigilancia, pues en el 2015 presentaron un problema que todavía no se ha resuelto a pesar de que se denunció y se encuentra en procesos legales. Son más de cuatro años que la falta de estos recursos afecta su calidad de vida:

Yo vivo en la calera, trabajé en el 2014 y 2015 con el proyecto, en actividades de compensación, enfocadas principalmente a la educación ambiental. El acueducto nunca había tenido el acercamiento a pesar de que hace más de 35 años pasaron un tubo de 3x50 debajo de sus terrenos. Pasaron como Pedro por su casa. Sin un permiso. Necesitaban para el proyecto alguien que viviera en la zona, yo me postule, pero cuando iba a hablar con las personas afectadas el acueducto se enojaba muchísimo. (ESC03).

\section{Discusión}

Se logra identificar por medio de la revisión literaria, de las entrevistas y noticias, algunos problemas inherentes al sistema Chingaza, los cuales de manera global se clasifican en: fauna, flora, económica y social.

Respecto a la fauna se logra observar que se presenta un fuerte papel histórico, como se puede observar con el oso de anteojos, una especie que sufrió de una caza indiscriminada, lo que llevó a la especie a ser catalogada en peligro de extinción en la resolución de 0192 de 2014 y 1912 de 2017 del Ministerio de ambiente y Desarrollo sostenible. La Unión Internacional para la Conservación de la Naturaleza indica que la principal amenaza es la pérdida de su hábitat por la expansión de la frontera agrícola, limitándose el espacio, por este motivo, las políticas emitidas por la CAR están enfocadas en la recuperación y mantenimiento del hábitat (Corporación Autónoma Regional de Cundinamarca, 2018). Se puede concluir que existe un pasivo ambiental en la fauna, pero que no puede ser asociado al sistema sino a los pobladores.

Es de asumir que la construcción del sistema Chingaza en el corto plazo generó una perturbación en su entorno y en el hábitat de diversas especies que todavía permanece, aunque se ha visto un proceso de recuperación de la zona por parte del Estado, la construcción de la represa de Chuza genera serias perturbaciones debido a que eliminó una porción de suelo que era de alta viabilidad para la recuperación del hábitat.

Un apartado que no se analizó a profundidad por la falta de información pero que tiene un impacto significativo, es la introducción de especies nuevas, específicamente el de aves migratorias atraídas por el cuerpo de agua. Este apartado es importante profundizarlo en futuras investigaciones (Ruiz, Álvarez, Ramírez, \& Cruz, 2017).

Adicionalmente, otra de las problemáticas de la represa son las descargas de agua que generan vibración en el suelo y sonidos que perturban la vida silvestre, además de incrementar el caudal de la cuenca, donde pueden estar animales cerca 
mientras se da este procedimiento, lo que genera el riesgo de que sean arrastrados por el agua. Sobre la variable de fauna y el papel de la EAB-ESP; se llega a la conclusión de que esta ha generado un daño, el cual se mantiene, y de ser implementada la fase II, se incrementaría al limitar el hábitat de la fauna. Respecto a los proyectos que se identificaron por parte de la empresa, se enfocaron en el suministro de veterinarios y políticas educativas para la población. Estas se pueden llegar a considerar ineficientes, ya que se podrían denominar como preventivas, más no como recuperadoras de los daños, lo que puede producir con el tiempo mantenimiento o crecimiento, en dado caso de no añadir más proyectos.

Después de analizar la flora, al igual que la fauna, se generó una perturbación previa a la construcción del sistema Chingaza, ya existían unos daños considerables que con la construcción del sistema llevó a la pérdida de más vegetación. La empresa EAB-ESP a pesar de tener unos daños en la zona, ha trabajado en procesos de reforestación, no solo en el parque si no con las comunidades aledañas, lo que generó una recuperación desde 1969, un ejemplo es el establecimiento de corredores ambientales, por este motivo no se puede hablar de que no existe un pasivo ambiental en esta variable, porque se ha retribuido con el tiempo y ha implementado proyectos que han servido a la región para la recuperación de la vegetación típica de la zona, cumpliendo los objetivos de preservación y recuperación del ecosistema (Vargas, Insuasty, Rojas, Castro, \& Flórez, 2012).

Respecto a las variables sociales y económicas. La primera afectación hacia la población se generó al establecer la zona como una reserva natural, lo que produjo que varias familias se tuvieran que movilizar, y la segunda, se da por la compra de EAB-ESP de terrenos para la construcción del sistema Chingaza. En este se da un pasivo ambiental de acuerdo con lo hallado en las entrevistas, dado que unos hogares de la Candelaria han tenido que incurrir en el pago de un mayor número de impuestos por terrenos que no son de su utilización, pero el que ha tenido la mayor repercusión es el acaparamiento del agua la cual ha limitado su utilización a diferentes grupos de la sociedad, principalmente pertenecientes a Cundinamarca y Meta.

Se limita la utilización del recurso a los habitantes al absorber gran parte del caudal, esta problemática se agrava en las épocas de sequias debido que se da prioridad al abastecimiento del sistema, vulnerando sus derechos, la empresa de acueducto ha implementado programas para el aprovechamiento de las lluvias para ser la principal forma de acceder al recurso. Esto genera varios problemas debido a que estas no son constantes. Los hogares de la zona que previo al establecimiento de la represa podían acceder con facilidad al agua, ahora no lo pueden hacer, o lo hacen de una manera parcial que no logra satisfacer sus necesidades. Esto enfrentó a las familias a la situación de migración o el adquirir un punto de agua el cual tenía unos costos muy elevados que muchas familias no podían permitirse. En el diseño del sistema estos hogares debieron ser tenidos en cuenta para establecer un sistema de tuberías para su beneficio.

Los hogares que no pueden acceder al agua se enfrentan a círculos viciosos de pobreza, debido a que la mayoría tenía como fuente de ingresos actividades agrícolas y ganaderas que, por políticas del parque y por la limitación al acceso del agua, disminuyeron, desencadenando disminuciones en la calidad de vida al tener menores ingresos. Aunque por parte del Estado se dieron incentivos económicos para migrar a prácticas más sostenibles de cultivos y la implementación de proyectos de turismo ambiental, el cual ha sido apoyado por la empresa EAB-ESP, no todas las familias se benefician de estos.

\section{Conclusión}

Después de analizar el papel de la población se encuentra una disyuntiva; innegablemente los asentamientos y el crecimiento de la sociedad impacta directamente en el ambiente de una manera negativa, pues se necesita una mayor cantidad de recursos para mantener una calidad de vida que al pasar de los años se ha compuesto de más variables. Los individuos de Colombia tienen una tendencia migratoria a las capitales de los departamentos y de la nación, un ejemplo de este comportamiento es Bogotá, ciudad que posee la mayor concentración de habitantes con 7.743.955, que corresponde al 15,37\% del total de la población del país. Colombia, al tener una sociedad aglomerada en varios puntos, genera la necesidad de creación de megaproyectos para satisfacer las necesidades básicas. Este tipo de obras tienen impactos ambientales a corto y largo 
plazo, en el caso del agua se generan sistemas de centralización del recurso debido al comportamiento de las personas, esto produce que los habitantes de las zonas rurales tengan dificultades para acceder, ya que las cuencas de los ríos se controlan o se desvian para satisfacer al común denominador (Ministerio de agua y saneamiento básico, 2018).

De los municipios que proveen los sistemas hídricos del país, el 58\% de estos no reciben agua apta para el consumo humano debido a la falta de infraestructura. Este indicador logra observar cómo una gran parte de la población de Colombia no puede acceder y está desprotegida (Ministerio de agua y saneamiento básico, 2018).

A pesar de que las obras se desarrollan para satisfacer las necesidades de una población mayor, uno de los puntos que se trató en el presente estudio fue la insatisfacción de las sociedades cercanas al no tener la opción del voto. Pero al ser proyectos que impactan en gran parte de la población, las votaciones deberían incluir tanto a los beneficiarios como los perjudicados. Es importante desarrollar estudios sobre las comunidades para poder brindar alternativas que les compense de una manera adecuada, y no tengan que pasar años en procesos de demandas para poder recuperar sus derechos y calidad de vida, los cuales, en algunos casos, no cuentan con la experticia jurídica para exigir sus derechos.

Respecto al papel de las entidades de control y las leyes para regular los temas ambientales, se identifica que son claras, pero no específicas, y enfocadas principalmente a la prevención. Esto es bueno, pero en muchos casos estas surgen tras la aparición de problemas ambientales. Un ejemplo es el sistema Chingaza que se implementó previo a la creación de las políticas, y que a medida que han pasado los años se ha ajustado a estas. Es de suma importancia que el Estado genere regulaciones más fuertes para que las industrias del país tomen prácticas más sustentables, además de generar procesos más fuertes en vigilancia, un sistema de auditorías y de comprobación de la información evitaría el surgimiento de pasivos ambientales.

$\mathrm{Al}$ analizar el ecoturismo se plantea como una práctica que ayuda a las familias de las zonas aledañas de reserva a conseguir una fuente de recursos, pero es importante dar vigilancia y medir los impactos causados por el turismo, generando controles de ingresos, ya que estas poblaciones realizan actividades de paisajismo y recorridos guiados, los cuales podrían estar pasando por corredores biológicos y haciendo una afectación en los ecosistemas, tales como pisar accidentalmente un frailejón o tomar muestras de plantas para enseñar a los visitantes, lo cual implicaría un proceso de recuperación de varios años.

Cada actividad económica implementada en un ecosistema requiere de un plan de monitoreo previo, durante y posterior a la finalización, ya que por básicos o complejos que sean sus procesos de producción genera alteraciones en el ambiente. La ausencia de análisis de impacto evidencia que hay una carencia de herramientas de control y monitoreo de los factores bióticos y abióticos, demostrando una ineficiencia en la administración, ya que no se cumplen las medidas de protección tal y como se decretan. Se demuestra que estas son aplicadas de una manera parcial, algunas veces privilegiando los intereses económicos potenciales sobre el cuidado de los recursos naturales.

\section{Referencias}

Herrera, C., Rodríguez, C., Tarquino, L., Varila, M., \& Martínez, C. (2015). Biodiversidad y servicios ecosistémicos en la alta montaña. Bogotá: Alcaldía mayor de Bogotá

Montoya A., \& Guzmán M. (2015). Gestión socioambiental y fortalecimiento institucional Somos Páramo Somos Comunidad.

Cárdenas, C. (2013). El fuego y el pastoreo en el páramo húmedo de Chingaza (Colombia): efectos de la perturbación y respuestas de la vegetación. (Tesis doctoral, Universidad Autónoma de Barcelona). Recuperado de https://tesisenred.net/han$\underline{\text { dle } / 10803 / 120219}$

Cubillos, C. (18 de noviembre1991). Guasca se desmorona. Recuperado de https://www.eltiempo.com/archivo/documento/MAM-193021

Empresa de Acueducto Alcantarillado y Aseo de Bogotá, \& Fundación para Desarrollo Sostenible Territorial. (2013). Caracterización y formulación participativa de estrategias, programas y proyectos para la recuperación, conservación y manejo sostenible de las microcuencas Quebrada Blanca y Siecha. Bogotá D.C.

Garavito, L., Gómez, D., \& Palacio, D. (2018). Gobernanza territorial en los páramos Chingaza y Sumapaz-Cruz Verde. Una comparación de sus principales actores y problemáticas. Perspectiva Geográfica, 23(1),20. https://doi. org $/ 10.19053 / 01233769.6703$

Morales M., Otero J., Van der Hammen T., Torres A., Cadena C. \& Rodríguez N., Franco C., Betan- 
courth J.C., Olaya E., P. E. y C. L. (2007). Atlas de páramos de Colombia. Instituto de Investigación de Recursos Biológicos Alexander von Humboldt. Bogotá D. C.

Núñez, G. (16 de febrero 2016). Chingaza Dos, un holocausto ambiental: Jiménez. Recuperado de https://diariolaeconomia.com/regiones-y-fronteras/ item/1937-chingaza-dos-un-holocausto-ambiental-jimenez.html

Pelayo, M., \& Gasca, J. (2019). Reconfiguración de un territorio hidrosocial tras la construcción de la presa El Cajón, en Nayarit. Región y sociedad, 31, e1201. https://doi.org/10.22198/rys2019/31/1201

Perdomo de Gómez, N., Lugo, S., \& Molano, R. (2011). Desarrollo jurisprudencial de la corte constitucional colombiana del principio de precaución frente a la libre empresa, en litigios ambientales. 1996 - 2016. (Tesis de maestría, Universidad del Cauca, Colombia) Recuperado de http://repositorio.unicauca.edu.co:8080/xmlui/handle/123456789/420

Pérez, M. (2018). La Justicia Ambiental como línea estratégica de la Economía Ecológica: ¿cómo evidenciar las injusticias ambientales? Gestión y Ambiente, 21(1supl), 57-68. https://doi. org/10.15446/ga.v21n1supl.75742

Pretelt, C. (2013). Análisis geoespacial de la ampliación del sistema Chingaza en la cuenca alta del río la playa, parque nacional natural Chingaza. Universidad Nacional de Colombia, Bogotá.

Redacción EL TIEMPO. (22 de julio de 1997. Río Guatiquía creció como nunca antes. Recuperado de https://www.eltiempo.com/archivo/documento/ MAM-718717

Redacción EL TIEMPO. (30 de abril 2008). Daños ambientales serán irremediables. Recuperado de https://www.eltiempo.com/archivo/documento/CMS$\underline{4135238}$

Redacción EL TIEMPO. (12 de enero 2019). Las amenazas que enfrenta Chingaza principal fuente de agua potable de Bogotá. Recuperado de https:// www.eltiempo.com/bogota/las-amenazas-que-enfrentachingaza-la-principal-fuente-de-agua-potable-de-bogota-313848

Ruiz, L., Álvarez, G., Ramírez, N., \& Cruz , B. (2017). Vulnerabilidad social y biológica ante el cambio climático en la Reserva de la Biosfera Selva El Ocote. Recuperado de www.ecosur.mx

Van der Hammen, T., Pabón, J., Gutiérrez, H., \& Alarcón, J. (2002). El Cambio Global y los ecosistemas de Alta Montaña de Colombia. Páramos y ecosistemas alto andinos de Colombia en condición hotspot y global climatic tensor. IDEAM.

Vargas, O., Insuasty, J., Rojas, O., Castro, P., \& Flórez, N. (2012). Programa para la restauración ecológica en áreas del parque nacional natural Chingaza. Recuperado de https://www.researchgate.net/publication/262935280_PROGRAMA_PARA_LA_RESTAURACION_ECOLOGICA_EN_AREAS_DEL_PARQUE_ NACIONAL NATURAL CHINGAZA

Vergara, C., \& Ortiz, D. (2016). Desarrollo sostenible: enfoques desde las ciencias económicas. APUNTES DEL CENES, 35(62), 15. https://doi. org/10.19053/22565779.4240

Viceministerio de Agua y saneamiento básico. (2018). Plan director de agua y saneamiento básico. Visión Estratégica 2018-2030, 1(4), 53. Recuperado de www.minvivienda.gov.co 